\title{
A Fractional Splitting Algorithm for Non-overlapping Domain Decomposition
}

\author{
Daoud S. Daoud and D. S. Subasi \\ Department of Mathematics, Eastern Mediterranean Univ., F amaqusta, North \\ Cyprus - Mersin 10 T urk ey \\ daoud.daoud@emu.edu.tr
}

\begin{abstract}
In this pap er w e studythe con vergenceof the non overlapping domain decomposition for solving large linear system arising from semi discretization of tw o dimensionalinitial value problem with homogeneous b oundary conditions, and solv ed by implicit time stepping using first and, tw o alternativ es of second order FS-methods. The interface values along the artificial boundary condition line are found using explicit forw ardEuler's method for the first order FS-method, and for the second order FS-method to use extrap olation pro cedure for each spatial variable individually. The solution by the non overlapping domain decomposition with FS-method is applicable to problems that requires the solution on non uniform meshes for each spatial variables which $\mathrm{w}$ ill unable us to use different time stepping over different sub domains, and with the p ossibilit yof extension to three dimensional problem.
\end{abstract}

\section{Introduction and Notation}

In domain decomposition the in terface boundary conditions for one or tw o dimensionalheat equations ha ve beenstudied by [D D DBLR1,Me1] and [Me2]. In tw odimensional problem its generally required iterative solution methods, with go od preconditioner,such as preconditioningconjugate gradien t methol [DDD1, Me1,Me2], to solve thesubproblems.

In literature one of the methods received great atten tioin solving two or three dimensional parabolic equation is the Fractionalsplitting method, FS-method, or dimensional splitting method [H1,GM1,KL1,S2]. The FS method w as firstlyin troducedby Godunov, 1959 [Go1], and no wits one of the classical methods [GM1,MG1, Y a1].

Our aim in this w orkis to presen t simplercriteria for defining in terface boundary condition for tw o dimensionalparabolic problem, which could be easily generalized to three dimensional problem. The approach is by incorp oratingin terface b oundary conditions defined for one dimensional problem with the one dimensional parabolic equation accomplished from FS-method along each spatial variable. The presen tedform of combination will reduce the cost of the algorithm and the storage requirements. This combination of the FS with the domain decomposition method has be en recen tly considered by Gaiffe, et.al, [GGL1], in order to provide 
a time discretization scheme to be different over each sub domains. In section 2, we present the model problem definition, the FS-method classifications, and their appropriate definition for the artificial boundary at the interface line for the 2-dimensional heat equation. In section 3 , we presented the theoretical aspects related to the error bound, stability and the convergence for the non overlapping FS-method of different order. The numerical experiment which support the convergence analysis, the comparison between the first and second order FS-method, and the relation with the number of subdomains are presented in section 4 .

\section{Preliminaries}

\subsection{The Model Problem}

In this work we consider the domain decomposition solution of linear two dimensional parabolic problem on the unit rectangular domain $\Omega=$ $(0,1) \times(0,1)$ with $0 \leq t \leq T$, given by

$$
\begin{array}{ll}
\frac{\partial u}{\partial t}=-\frac{\partial^{2} u}{\partial x^{2}}-\frac{\partial^{2} u}{\partial y^{2}} & \text { on } \Omega \\
\text { B.C. }: u(x, y, t)=0 & \text { on } \Gamma=\partial \Omega, \text { and } \\
\text { I.C. }: u(x, y, 0)=u_{0}(x, y) & \text { on } \Omega \cup \Gamma
\end{array}
$$

Let $h=\frac{1}{N+1}$, be the mesh spacing for the spatial variables $x$ and $y$, and let $\Omega_{h}$ be the spatial grid domain defined by $\Omega_{h}=\left\{(x, y) \mid x_{i}=i h, y_{j}=\right.$ $j h, 1 \leq i, j \leq N\}$.

The grid functions $\boldsymbol{u}_{i, j}^{m}$ representing the discrete solution over $\Omega_{h}$, represented by vectors in $\mathbf{R}^{N \times N}$, with natural row ordering for the grid functions at time $t_{m}$.

The spatial operator $-\frac{\partial^{2}}{\partial x^{2}}-\frac{\partial^{2}}{\partial y^{2}}$, is approximated by the standard second order finite difference discretization ( central difference) over $\Omega_{h}$, and after assembling the unknowns it will leads to a semi discrete system of first order differential equations [LP1].

$$
\frac{d u}{d t}=-A u
$$

where $A$ is the global matrix of coefficients presented with 5 non zero diagonal entries. The exact solution of (1) satisfies the two term recurrence relation involving the matrix exponential function of the matrix $A$ given by

$$
u(t+\delta t)=e^{-\delta t A} u(t)
$$

where $\delta t$ is the time step.

Due to the existence of the constituent splitting of $A$ as $A=A_{1}+A_{2}$, which they (i.e. $A_{1}$ and $A_{2}$ ) are of tridiagonal entries. Then $e^{A} \simeq e^{A_{1}+A_{2}}$ will be considered in terms of $e^{A_{1}}$ and $e^{A_{2}}$ as induced by the fractional step method FS [MPR1,Ya1,GM1,MG1].

The fractional splitting method FS due to Yanenko [Ya1], is based on simple splitting of the spatial operator along the spatial variables. 
The first step tow ardssolving the model problem (1) is to consider the FS method, whic hcan be expressed as follows:

$$
\begin{array}{ll}
\frac{1}{2} u_{t}=u_{x x} & \text { over }\left[t_{m}, t_{m}+\frac{\delta t}{2}\right], \quad \text { and } \\
\frac{1}{2} u_{t}=u_{y y} & \text { over }\left[t_{m}+\frac{\delta t}{2}, t_{m+1}\right] .
\end{array}
$$

The solution for (4), is by theuse of the semi discretization, with resp ect to the spatial variable, such that the recursiv esolution for $\boldsymbol{u}_{1}$, and $\boldsymbol{u}_{2}$ over the time in terval $\left.t_{m}, t_{m+1}\right]$, is given by

$$
\begin{aligned}
& u\left(t_{m}+\frac{\delta t}{2}\right)=e^{-\delta t A_{1}} u\left(t_{m}\right), \\
& u\left(t_{m}+\delta t\right)=e^{-\delta t A_{2}} u\left(t_{m}+\frac{\delta t}{2}\right),
\end{aligned}
$$

and the solution of (5), will b e appro ximatedısing $\mathrm{P}$ ade'appro ximation for $e^{-\delta t A_{i}}$.

The FS methods are classified according to the order of the exp onen tial appro ximationeither as a locallysecond order i.e. $O\left(\delta t^{2}\right)$ represen tedby $(1,0)$ pade' appro ximationor equiv alen tlythe bac kw ardEuler's scheme giv en by:

$$
e^{-\delta t A_{i}} \simeq\left(I+\delta t A_{i}\right)^{-1}+O\left(\delta t^{2}\right)
$$

or as a locallythird order i.e. $O\left(\delta t^{3}\right)$ whic $\mathrm{h}$ represen ted by $(1,1) \mathrm{P}$ arie $\mathrm{e}^{2}$ pro ximationor equiv alen tly the Crankicolson(C.N.)-scheme [H1,MPR1], giv en by

$$
e^{-\delta t A_{i}} \simeq\left(I+\frac{\delta t}{2} A_{i}\right)^{-1}\left(I-\frac{\delta t}{2} A_{i}\right)+O\left(\delta t^{3}\right)
$$

The in troduced classification is consequeted from the order of the pow er series represen tation of $e^{\delta t A_{i}}$, and also from the commutativit y of $A_{1}$ and $A_{2}$ e.g. [H1 ,MPR]. F or the first order FS-method (3) is rewritten as follows;

$$
e^{-\delta t A} \simeq e^{-\delta t A_{1}} e^{-\delta t A_{2}}
$$

In a non commutativ ecase, the highest possible order to consider for $e^{\delta t A_{i}}$, is the first order [H1,KL1,MPR1,S1,Sw1], and to achiev e a higher order pow er series fore ${ }^{\delta t A}$, Strang [S1] presen $\mathrm{t}$ analgorithm to provide a third order appro ximationfor $e^{\delta t A}$ achiev edby the following splitting of $A \mathrm{~g}$ iven by

$$
e^{-\delta t A} \simeq e^{-\frac{\delta t}{2} A_{1}} e^{-\delta t A_{2}} e^{-\frac{\delta t}{2} A_{1}},
$$

when, a locally, thirdorder appro ximationsfor $e^{-\frac{\delta t}{2} A_{1}}$ and $e^{-\delta t A_{2}}$ are considered [MPR1].

A further prop osalto provide a second order presen tationby means of po wer series of $e^{-\delta t A_{i}}$, is due to $\mathrm{Sw}$ ayn€Sw1], giv en by

$$
e^{-\delta t A}=\frac{1}{2}\left[e^{-\delta t A_{1}} e^{-\delta t A_{2}}+e^{-\delta t A_{2}} e^{-\delta t A_{1}}\right] .
$$

\section{Interface Boundary Condition}

\subsection{First Order FS-Method}

F or the first order FS method w e willconsider the definition of the interface boundary conditions by the F orw ardEuler's appro ximationdue 
to Dawson, et, al [DDD1] for each spatial variable, along the interface line is given by the following algorithm:

\section{Algorithm 1:}

1. Let $\bar{x}(=p h)$ for $1<p<N$, be an interface point for the spatial variable $x$, such that the domain $\Omega$ is then split into $\Omega_{\bar{x}, 1}=\left\{\left(x_{i}, y\right) \mid 0<\right.$ $\left.x_{i}<\bar{x}, 0<y<1\right\}$, and $\Omega_{\bar{x}, 2}=\left\{\left(x_{i}, y\right) \mid \bar{x}<x_{i}<1,0<y<1\right\}$. At $t=t_{m}+\frac{1}{2} \delta t$, the interface boundary condition along the interface line $\left(\bar{x}, y_{j}\right), j=1, \ldots, N$, is given by

$$
\boldsymbol{u}_{\bar{x}, y_{j}}^{m+\frac{1}{2}}=\boldsymbol{u}_{p, j}^{m+\frac{1}{2}}=r \boldsymbol{u}_{p-1, j}^{m}+(1-2 r) \boldsymbol{u}_{p, j}^{m}+r \boldsymbol{u}_{p+1, j}^{m}, \quad j=1, \ldots, N,
$$

where $r=\frac{\delta t}{H_{x}}$, and $H_{x}$ is the integral multiple of the mesh spacing $h_{x}$ with respect to the spatial variable $x$. The intermediate solution $\boldsymbol{u}^{m+\frac{1}{2}}$ is then given by solving $\left(I+\delta t A_{1}\right) \boldsymbol{u}^{m+\frac{1}{2}}=\boldsymbol{u}^{m}$, over $\Omega_{\bar{x}, 1}$ and $\Omega_{\bar{x}, 2}$ respectively using the backward Euler's scheme.

2. Let $\bar{y}(=q h)$ for $1<q<N$, be an interface point for the spatial variable $y$, such that the domain $\Omega$ is then split as follows: $\Omega_{\bar{y}, 1}=$ $\left\{\left(x, y_{i}\right) \mid 0<x<1,0<y<\bar{y}\right\}$, and $\Omega_{\bar{y}, 2}=\left\{\left(x, y_{i}\right) \mid 0<x<1, \bar{y}<\right.$ $y<1\}$. At $t=t_{m+1}$ the interface boundary conditions along the interface line $\left(x_{i}, \bar{y}\right)$ for $i=1, \ldots, N$, is given by

$$
\boldsymbol{u}_{x_{i}, \bar{y}}^{m+1}=\boldsymbol{u}_{i, q}^{m+1}=r \boldsymbol{u}_{i, q+1}^{m+\frac{1}{2}}+(1-2 r) \boldsymbol{u}_{i, q}^{m+\frac{1}{2}}+r \boldsymbol{u}_{i, q-1}^{m+\frac{1}{2}}, \quad i=1, \ldots, N,
$$

where $r=\frac{\delta t}{H_{y}^{2}}$ and $H_{y}$ is the integral multiple of the mesh spacing $h_{y}$, with respect to the spatial variable $y$.

Then solve for $\boldsymbol{u}^{m+1}$ the system $\left(I+\delta \boldsymbol{t} A_{2}\right) \boldsymbol{u}^{m+1}=\boldsymbol{u}^{m+\frac{1}{2}}$, over $\Omega_{\bar{y}, 1}$ and $\Omega_{\bar{y}, 2}$.

In our analysis we will consider the maximum norm and define the max norm of the error at the interface boundary points by

$$
\left\|\tilde{E}_{x_{p}}^{m}\right\|_{j}=\max _{j}\left|U_{p, j}^{m}-u_{p, j}^{m}\right| \text {, and }\left\|\tilde{E}_{y_{p}}^{m}\right\|_{i}=\max _{i}\left|U_{i, q}^{m}-u_{i, q}^{m}\right|,
$$

where $x_{p}$ and $y_{p}$ are the interface points for $x$ and $y$ respectively, and $\tilde{E}^{m}=\max \left\{\tilde{E}_{x_{p}}^{m}, \tilde{E}_{y_{p}}^{m}\right\}$.

Lemma 1. If $A_{1}$ and $A_{2}$ are symmetric and positive definite matrices, then the first order-FS method is stable in maximum norm independent of $h$ and $\delta t$.

The following theorem due to Mathew, et al [MPR1], concerns the truncation error of the first order-FS method.

Theorem 1. The first order-FS method approximate solution $W^{m+1}$ of $(I+\delta \boldsymbol{t} A) \boldsymbol{u}^{m+1}=\boldsymbol{u}^{m}$ solves

$$
(I+\delta t A) W^{m+1}+\left(\delta t^{2} \sum_{1 \leq k_{1}, k_{2} \leq 2} A_{k_{1}} A_{k_{2}}\right) W^{m+1}=W^{m} .
$$

The local truncation error $T_{F S 1}$ of the first orderFS method has the terms $T_{F S 1}=T+\left(\delta t^{2} \sum_{1 \leq k_{1}, k_{2} \leq 2} A_{k_{1}} A_{k_{2}}\right) u^{m+1}$ in addition to the terms in the local truncation error $T$ when exact solvers are used. Here $u^{m+1}$ is the exact solution of the parabolic equation (1). 
Proof. For the proof see [MPR1].

From the incorporation of the interface boundary conditions given by Algorithm 1, with the FS method, the error bound estimates for the overall error $E^{m+1}=u^{m+1}-W^{m+1}$ at time $t=t_{m+1}$, is given by the following theorem.

Theorem 2. If $A_{1}$, and $A_{2}$ are symmetric and positive semi definite matrices, then the error bound for algorithm 1, satisfies the relation $\left\|E^{m+1}\right\| \leq\left\|E^{m}\right\|+O\left(\delta t^{2}\right)$, and hence algorithm 1, is stable.

The error at $\left(x_{i}, y_{j}, t_{m}\right)$ due to the utilization of Dawson's algorithm in the first order FS-method subject to non-overlapping subdomains is given by.

Theorem 3. If $\frac{1}{2}\left|\frac{\partial^{2} u}{\partial t^{2}}\right|, \frac{1}{12}\left|\frac{\partial^{4} u}{\partial x^{4}}\right|$, and $\frac{1}{12}\left|\frac{\partial^{4} u}{\partial y^{4}}\right|$ are bounded by constant $C$ on $\Omega \times T$, and $\delta t \leq \max \left\{\frac{H_{y}^{2}}{2}, \frac{H_{x}^{2}}{2}\right\}$, then

$$
\max _{i, j, m}\left|u_{i, j}^{m}-W_{i, j}^{m}\right| \leq \frac{C^{2}}{16}\left[h_{x}^{2}+h_{y}^{2}+H_{x}^{3}+H_{y}^{3}+\delta t^{2}\right],
$$

where $H_{x}$, and $H_{y}$ are he integral multiplies of $h_{x}$, and $h_{y}$ respectively.

Proof. Consider the solution method given in (5) for each spatial variable over time interval of length $\frac{\delta t}{2}$, with non overlapping two subdomains, then;

$$
\begin{aligned}
& \max _{i, j, m}\left|u_{i, j}^{m}-W_{i, j}^{m}\right| \leq\left(\max _{j, m} \mid u\left(x_{i}, y_{j}, t^{m}\right)-W\left(x_{i}, y_{j}, t^{m}\right)\right) \\
&\left(\max _{i, m}\left|u\left(x_{i}, y_{j}, t^{m}\right)-W\left(x_{i}, y_{j}, t^{m}\right)\right|\right) .
\end{aligned}
$$

For the heat equation defined by $E_{i, j}^{m+1}$, with the homogeneous boundary conditions, and the artificial boundary conditions at the interface line (Algorithm 1), then for the spatial variable $x$, we have

$$
\max _{i, m}\left|u\left(x_{i}, y_{j}, t^{m}\right)-W\left(x_{i}, y_{j}, t^{m}\right)\right| \leq C\left[\frac{1}{8}\left(\frac{\delta t}{2}+h_{x}^{2}\right)+\frac{1}{4} H_{x}\left(H_{x}^{2}+\frac{\delta t}{2}\right)\right] .
$$

Similarly for the spatial variable $y$,

$$
\max _{j, m}\left|u\left(x_{i}, y_{j}, t^{m}\right)-W\left(x_{i}, y_{j}, t^{m}\right)\right| \leq C\left[\frac{1}{8}\left(\frac{\delta t}{2}+h_{y}^{2}\right)+\frac{1}{4} H_{y}\left(H_{y}^{2}+\frac{\delta t}{2}\right)\right]
$$

By substituting (12), and (13), in (11),

$$
\begin{aligned}
\max _{i, j, m}\left|u_{i, j}^{m}-W_{i, j}^{m}\right| \leq & C\left[\frac{1}{8}\left(\frac{\delta t}{2}+h_{x}^{2}\right)+\frac{1}{4} H_{x}\left(H_{x}^{2}+\frac{\delta t}{2}\right)\right] \\
& C\left[\frac{1}{8}\left(\frac{\delta t}{2}+h_{y}^{2}\right)+\frac{1}{4} H_{y}\left(H_{y}^{2}+\frac{\delta t}{2}\right)\right] .
\end{aligned}
$$

After performing the desired product for the terms in the last equation and summing up the terms of the same order the desired results is obtained given as follows,

$$
\max _{i, j, m}\left|u_{i, j}^{m}-W_{i, j}^{m}\right| \leq \frac{C^{2}}{16}\left[h_{x}^{2}+h_{y}^{2}+H_{x}^{3}+H_{y}^{3}+\delta t^{2}\right] .
$$




\subsection{Interface Boundary Condition- Second Order FS-Method}

The globally second order FS-method can be achieved using Strang's splitting for $A, A=\frac{1}{2} A_{1}+A_{2}+\frac{1}{2} A_{1}$, such that

$$
e^{-\delta t A} \simeq e^{-\frac{\delta t}{2} A_{1}} e^{-\delta t A_{2}} e^{-\frac{\delta t}{2} A_{1}}
$$

[KL1,MPR1,S1,S2].

The exponential term $e^{-\delta t B},\left(\delta t B=\frac{\delta t}{2} A_{1}\right.$, or $\left.\delta t A_{2}\right)$ in (16), are approximated by $(1,1)$ Pade' approximation of locally third order $O\left(\delta t^{3}\right)$, denoted by $R_{1,1}(\delta t B)$, given by

$$
e^{-\delta t B} \simeq R_{1,1}(-\delta t B)=\left(I+\frac{\delta t}{2} B\right)^{-1}\left(I-\frac{\delta t}{2} B\right)+O\left(\delta t^{3}\right),
$$

or equivalently the C.N. scheme [H1,MPR1].

The solution $u(t+\delta t)$ of (3), is evaluated by using an intermediate solution vectors, internal estimation vectors, $w$ and $v$, as follows;

$$
\boldsymbol{u}(\boldsymbol{t}+\delta \boldsymbol{t})=e^{-\frac{\delta t}{2} A_{1}} \boldsymbol{w}, \text { where } \boldsymbol{w}=e^{-\delta t A_{2}} \boldsymbol{v}, \text { and } \boldsymbol{v}=e^{-\frac{\delta t}{2} A_{1}} \boldsymbol{u}
$$

In addition to the above scheme (18), Swayne [Sw1] presented a further type of second order approximation to $e^{-\delta t A}$ given by :

$$
e^{-\delta t A}=\frac{1}{2}\left[e^{-\delta t A_{1}} e^{-\delta t A_{2}}+e^{-\delta t A_{2}} e^{-\delta t A_{1}}\right],
$$

such that the solution $u(t+\delta t)$ of (3), is given by;

$$
u(t+\delta t)=\frac{1}{2}\left[u^{(1)}(t+\delta t)+u^{(2)}(t+\delta t)\right] .
$$

To provide an interface boundary condition $u_{I}^{m}$, for each spatial variables, with second order accurate in time i.e. with leading error term $O\left(\delta t^{3}\right)$ we considered an extrapolation for $\boldsymbol{u}^{m-\gamma \delta t}$ and $\boldsymbol{u}^{m-\beta \delta t}$, for $0<\gamma \leq 1$ and $1<\beta \leq 2$, given by

$$
(\beta-\gamma) u_{I}^{m}=\beta u^{m-\gamma \delta t}-\gamma \boldsymbol{u}^{m-\beta \delta t}+O\left(\delta t^{3}\right),
$$

for $m \geq 2, \gamma \leq 1$, and $\beta=2$, then (21) is given by

$$
u_{I}^{m}=2 u^{m-1}-u^{m-2}+O\left(\delta t^{3}\right),
$$

where $u_{I}^{m}=u_{p, j}^{m}$ or $u_{I}^{m}=u_{i, q}^{m}$ are the interface boundary conditions for the spatial variables $x$ or $y$ respectively. The above approach has also a flexibility to be used even for estimating the interface boundary condition for a higher space dimension, and its a stable scheme for positive $\delta t$ [LM1]. The domain decomposition algorithm for (1), by the second order FS defined by Strang's splitting method with the boundary condition defined by (21) is given by the following algorithm:

\section{Algorithm 2:}

Let $\boldsymbol{u}^{1}$ be the solution from the second order FS method or any second order method in time e.g. C.N., and $\boldsymbol{u}^{0}$ be the initial condition of (1), then for $m \geq 2$, and with the interface boundary condition given by (21) according to each spatial variable. 
1. Let $\left(\bar{x}, y_{j}\right), \bar{x}=p h, 1<p<N, j=1, \ldots, N$ be interface points for the spatial variable $x$ over $\left[t_{m}, t_{m+\frac{1}{2}}\right]$, and solve for $v, v=e^{-\frac{\delta t}{2} A_{1}} u$.

2. Let $\left(x_{j}, \bar{y}\right), \bar{y}=q h, 1<q<N, i=1, \ldots, N$ be interface points for the spatial variable $y$ over $\left[t_{m}, t_{m}+\delta t\right]$, and solve for $w, w=e^{-\delta t A_{2}} v$.

3. Solve for $u, u=e^{-\frac{\delta t}{2} A_{1}} w$ over $\left[t_{m}+\frac{\delta t}{2}, t_{m}+\delta t\right]$, with the interface boundary conditions given by (21) with respect to the spatial variable $x$, i.e. at the interface points $\left(\bar{x}, y_{j}\right)$.

The domain decomposition algorithm for (1), by the second order FS defined using Swayne's splitting method with the boundary condition defined by (21) is given by the following algorithm:

\section{Algorithm 3:}

Let $u^{1}$ be the solution from the second order FS method or any second order method in time e.g. C.N., and $\boldsymbol{u}^{0}$ be the initial condition of (1), then for $m \geq 2$,

1. Let $\left(\bar{x}, y_{j}\right), \bar{x}=p h, 1<p<N, j=1, \ldots, N$ be interface points for the spatial variable $x$ over $\left[t_{m-1}, t_{m}\right]$, with interface boundary conditions given by (21), at $t_{m}$, and solve for $\boldsymbol{u}^{*}, \boldsymbol{u}^{*}=e^{-\delta t A_{1}} u$.

2. Let $\left(x_{i}, \bar{y}\right), \bar{y}=q h, 1<q<N, i=1, \ldots, N$ be interface points for the spatial variable $y$ over $\left[t_{m-1}, t_{m}\right]$ with interface boundary conditions given by (21), at $\boldsymbol{t}_{m}$, and solve for $\boldsymbol{w}, \boldsymbol{w}=e^{-\delta t A_{2}} \boldsymbol{u}^{*},\left(\boldsymbol{u}^{(1)}=\boldsymbol{w}\right)$.

3. Let $\left(x_{i}, \bar{y}\right), \bar{y}=q h, 1<q<N, i=1, \ldots, N$ be interface points for the spatial variable $y$ over $\left[t_{m-1}, t_{m}\right]$, with interface boundary conditions given by (21), at $\boldsymbol{t}_{m}$, and solve for $\tilde{\boldsymbol{u}}, \tilde{\boldsymbol{u}}=e^{-\delta t A_{2}} \boldsymbol{u}$.

4. Let $\left(\bar{x}, y_{j}\right), \bar{x}=p h, 1<p<N, j=1, \ldots, N$ be interface points for the spatial variable $x$ over $\left[t_{m-1}, t_{m}\right]$ with interface boundary conditions given by (21), at $t_{m}$, and solve for $v, v=e^{-\delta t A_{2}} \tilde{u},\left(u^{(2)}=v\right)$. Then $u(t+\delta t)=\frac{1}{2}\left(u^{(1)}+u^{(2)}\right)$.

The stability of the second order FS method defined by Strang's splitting [MPR1], and the Swayne's proposal governed by the stability of the rational approximation $R_{1,1}\left(\delta t A_{i}\right)$ for the exponent of the matrices $A_{1}$, and $A_{2}$, which is due to the stability of the C.N.-Scheme, [H1,MPR1,Sw1]. The following theorem concerns the truncation error for the second order FS-splitting.

Theorem 4. The second order-FS method approximates solution $W^{m+1}$ of

$$
R_{1,1}(\delta t A) u^{m+1}=u^{m}
$$

by Strang's Splitting, solves

$$
\begin{gathered}
R_{1,1}\left(\frac{\delta t}{2} A_{1}\right) R_{1,1}\left(\delta t A_{2}\right) R_{1,1}\left(\frac{\delta t}{2} A_{1}\right) W^{m+1}+ \\
\left(\delta t^{3} \sum_{j, 1 \leq k_{1}, k_{2}, k_{3} \leq 2} \tau_{j} A_{k_{1}} A_{k_{2}} A_{k_{3}}\right) W^{m+1}=W^{m}
\end{gathered}
$$

and by Swayne's proposal solves 


$$
\begin{gathered}
\frac{1}{2}\left(R_{1,1}\left(\delta t A_{1}\right) R_{1,1}\left(\delta t A_{2}\right)+R_{1,1}\left(\delta t A_{2}\right) R_{1,1}\left(\delta t A_{1}\right)\right) W^{m+1}+ \\
\left(\delta t^{3} \sum_{j, 1 \leq k_{1}, k_{2}, k_{3} \leq 2} \rho_{j} A_{k_{1}} A_{k_{2}} A_{k_{3}}\right) W^{m+1}=W^{m}
\end{gathered}
$$

The local truncation error $T_{F S}$ of the second order FS-method has the following terms in addition to the terms in the local error $T$ when exact solvers are used,

$$
T_{F S}(\text { strang })=T+\left(\delta t^{3} \sum_{j, 1 \leq k_{1}, k_{2}, k_{3} \leq 2} \tau_{j} A_{k_{1}} A_{k_{2}} A_{k_{3}}\right) u^{m+1}
$$

and

$$
T_{F S}(\text { swayne })=T+\left(\delta t^{3} \sum_{j, 1 \leq k_{1}, k_{2}, k_{3} \leq 2} \rho_{j} A_{k_{1}} A_{k_{2}} A_{k_{3}}\right) u^{m+1},
$$

corresponding to Strang's Splitting and Swayne's proposal, respectively. Here $\boldsymbol{u}^{m+1}$ is the exact solution of the parabolic equation (21).

Corollary 1. The maximum norm of the $O\left(\delta t^{3}\right)$ term, in $\left(e^{\delta t A}-e^{\frac{\delta t}{2} A_{1}} e^{\delta t A_{2}} e^{\frac{\delta t}{2} A_{1}}\right)$ by Strang's splitting is given by;

$$
\frac{O\left(\delta t^{3}\right)}{6}\left(30\left\|A_{1}\right\|^{2}\left\|A_{2}\right\|+20\left\|A_{1}\right\|\left\|A_{2}\right\|^{2}\right),
$$

and the maximum norm of the $O\left(\delta t^{3}\right)$, term $\left(e^{\delta t A}-\frac{1}{2}\left(e^{\delta t A_{1}} e^{\delta t A_{2}}+e^{\delta t A_{2}} e^{\delta t A_{1}}\right)\right)$, by Swayne's proposal is given by;

$$
O\left(\delta t^{3}\right)\left(2\left\|A_{1}\right\|^{2}\left\|A_{2}\right\|+2\left\|A_{2}\right\|^{2}\left\|A_{1}\right\|\right)
$$

\section{Numerical Experiments and Conclusion}

The proposed algorithms 1,2, and 3 are examined to solve the following two dimensional heat equation

$$
\frac{\partial u}{\partial t}=\frac{\partial^{2} u}{\partial x^{2}}+\frac{\partial^{2} u}{\partial y^{2}}, 0 \leq x, y \leq 1, t>0
$$

with boundary conditions $u(0, y, t)=u(1, y, t)=0,0 \leq y \leq 1$, and $u(x, 0, t)=u(x, 1, t)=0,0 \leq x \leq t>0$, and initial conditions $u(x, y, 0)=\sin (\pi x) \sin (\pi y), 0 \leq x, y \leq 1$. The standard central difference approximation was used for the discretization for each spatial variable $x$, and $y$, on uniform grid with $h=\frac{1}{100}$, and $\frac{1}{150}$, for the spatial variables $x$, and $y$, and the discretized problem is then solved over time intervals $t=0.1,0.2,0.5$, and $t=1$. The solution is then discussed according to different number of subdomains.

Figure 1 plots the maximum error by algorithms 1, 2, and 3, for 2, and 15 subdomains by mesh spacing $h=\frac{1}{150}$, over $t=[0,1]$.

The figure indicated that the error by the second order FS-algorithms, algorithms 2, and 3 has less error than the first order, and that is due to the order of splitting as referred in theorems 3, 4, and also due to 

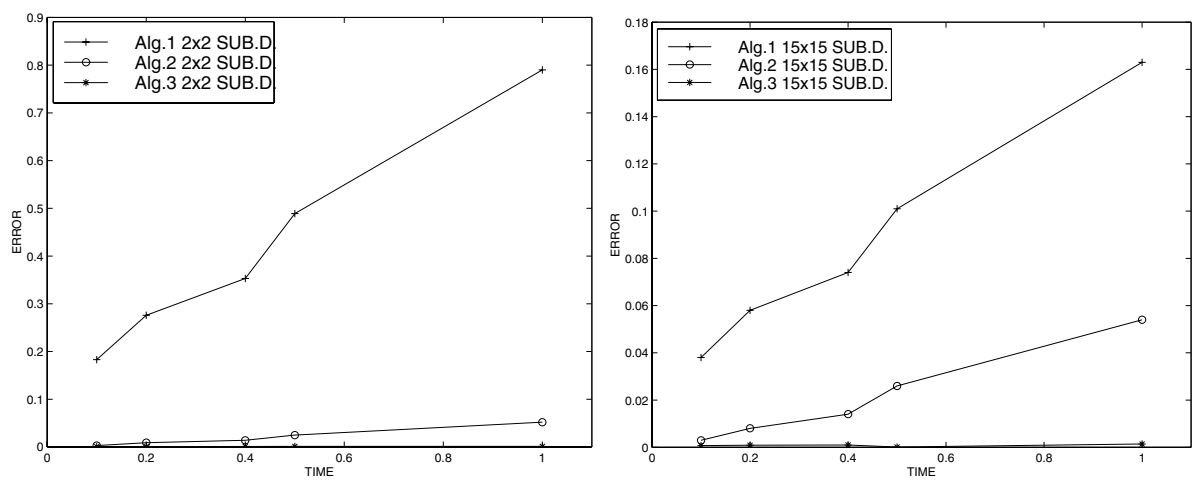

Fig. 1. The error by the FS-algorithms 1, 2, and 3, on the left for 2 subdomains and on the right for 15 subdomains, for $h=\frac{1}{150}$, over the time interval $[0,1]$.

the error at the artificial boundary at the interface predicted by the forward Euler's scheme which posses a restriction on the $\delta t$ as given in theorem 3, not like the case in the prediction of the interface for the second order FS-method. Also, the error by algorithm 3 produce the solution with less error than the solution by algorithm 2 which is due to corollary 1 of theorem 4 . Figure 2, shows the plots of maximum error by the algorithms 1 and 2, respectively over different time intervals $t=0.1,0.2,0.4,0.5$, and 1 , solved for different number of sub domains, we concluded that the algorithm of the first order FS-method depends on the number of the subdomains but on the contrary the second order FS-method does not depend on the number of the sub domains. The
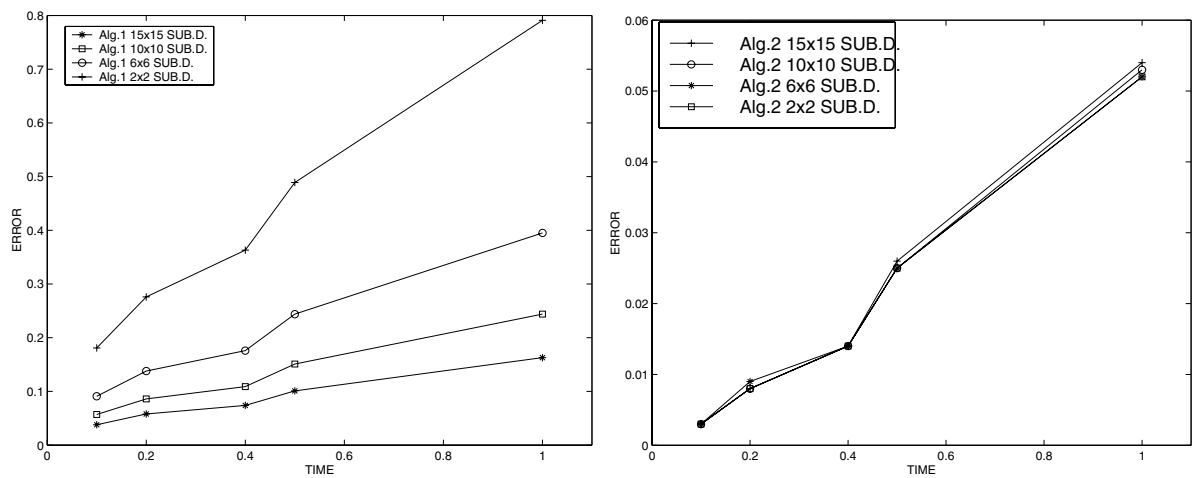

Fig. 2. The effect of the number of subdomains on the error of the first order FSalgorithm 1 on the left for and on the right for FS-algorithm 2 for $h=\frac{1}{150}$, over the time interval $[0,1]$. 

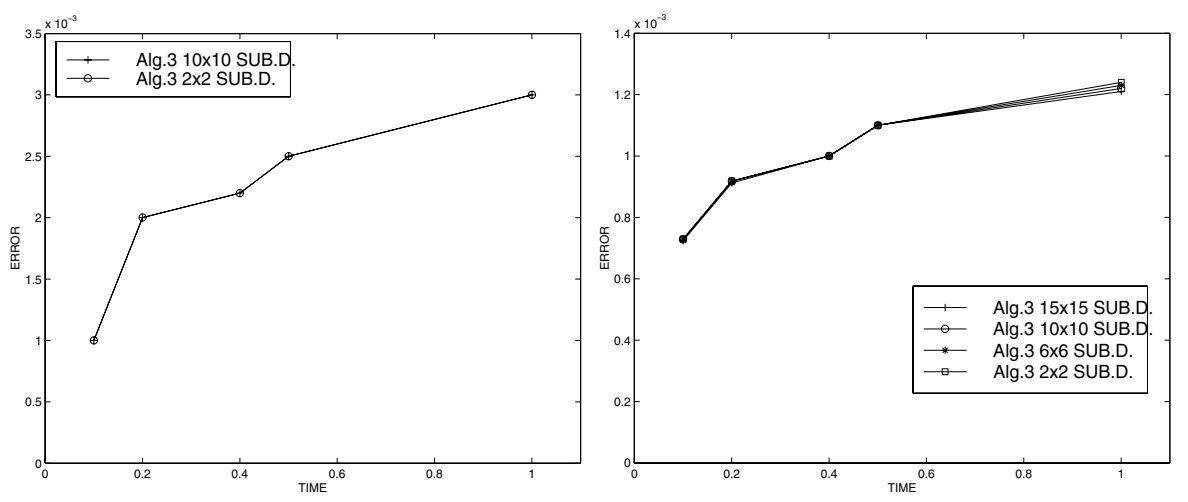

Fig. 3. The second order FS-algorithm 3, is independent on the number of the subdomains, on the right for $h=\frac{1}{150}$ and on the left for $h=\frac{1}{100}$ over the time interval $[0,1]$.

deterioration of the accuracy(error) in the domain splitting algorithm for larger time interval is mainly due to the truncation error in the discretization and the interface prediction of the boundary values at the artificial boundary of the interface. A similar conclusion is concluded by algorithm 3 as illustrated in figure 3 for different mesh spacing. For both types of splitting by algorithms 2, and 3 the numerical results demonstrates that the error from the solution of the model problem does not depend on the number of the sub domains, not like the case in the first order FS-method algorithm 1, as shown in fig(2).

The main features of the presented work are the following points:

1. The algorithm provide a solution of the original problem on subdomains and thus allows a refinement in time variable, and in $x y$-plane, according to each space variable independently and then solve over each subdomain for each space variable with independent mesh spacing. The algorithm is also extendable to three dimensional problem.

2. The second order FS- method, by Swayne's algorithm, by itself is a completely parallel algorithm called parallel alternating scheme [H1], according to each term in (19), and provides a further parallelism enhanced by the solution of the subproblem over a non overlapping subdomains according to each spatial variable.

\section{References}

[BLR1] Blum, H.,Lisky, S. and Ranacher, R.," A domain Splitting Algorithm for Parabolic Problems," Computing, 49:11-23, 1992.

[DDD1] Dawson,C.N.,Du, Q., and Dupont, D.F." A finite Difference Domain Decomposition Algorithm for Numerical Solution of the Heat Equation," Mathematics of Computation, 57:63-71, 1991. 
[GGL1] Gaiffe, S., Glowinski,R. and Lemonnier, P. " Domain Decomposition and Splitting Methods for Parabolic Problems via a Mixed Formulation", The $12^{t h}$ International Conference on Domain Cecomposition, Chiba -Japan,1999.

[GM1] Gourlay, A. R. and A.R. Mitchell," The Equivalence of Certain ADI and LOD Difference Methods," SIAM J. Numer. Anal.,6:37,1969.

[Go1] Goudnov, S. K.," Finite Difference Methods for Numerical Computation of Discontinuous Solutions of the Equations of Fluid Dynamics", Mat.Sbornik, 47:271-295, 1959.

[H1] Hundsdorfer, W.," Unconditional Convergence of Some CrankNicolson LOD Methods for Initial Boundary Value Problems," Mathematics Of Computation, 58(197):35-53, 1992.

[KL1] Khan, L.A., and P.L. Liu," An Operator-Splitting Algorithm for the Three-Dimensional Diffusion Equation," Numerical methods for Partial Differential Equations 11:617-624, 1995.

[LM1] Lawson, J. D. and Morris J, LI, " The Extrapolation of First Order Methods for Parabolic Partial Differential Equations. I," SIAM J. Numer. Anal. 15:1212-1224, 1978 .

[LP1] Lapidus, L., and Pinder, G.F.: " Numerical Solution of Partial Differential Equations in Science and Engineering", John Wiley, 1982.

[Me1] Meurant, G.A.," A Domain Decomposition Method for Parabolic Problems," Applied Numerical Mathematics, 8:427-441,1991.

[Me2] Meurant,G.A.," Numerical Experiments with Domain Decomposition Method for Parabolic Problems on Parallel Computers," $4^{\text {th }}$ "Int. Sypm. on Domain Decomposition methods for Partial Differential Equations," ed. Glowinski,R.,Kunetsov,Y.A., Meurant,G.A., ,Periaus, J., and Widlund,O.W., SIAM, PA., 394$408,1991$.

[MG1] Mitchell, A.R. and Griffiths, D.F.," The Fnite Difference Methods in Partial Differential Equations", John Wiley, 1980.

[MPR1] Mathew, T.P., Polyakov, P.L., Russo,G., and Wang, J.," Domain Decomposition Operator Splittings for the Solution of Parabolic Equations," SIAM J.Sci.Comput. 19:912-932, 1998.

[S1] Strang, G.," Accurate Partial Difference Methods I: Linear Cauchy Problems," Arch. Rational Mech. Anal., 12:392-402, 1963.

[S2] Strang,G.," On the construction and Comparison of Difference Schemes," SIAM J. Numer. Anal., 5:506-517, 1968.

[Sw1] Swayne, D.A.," Time Dependent Boundary and Interior Forcing in Locally One Dimensional Schemes," SIAM J.Sci.Stat.Comput, 8:755-767, 1987.

[Ya1] Yanenko, N.N.," The method of Fractional Steps; The Solution of Problems of Mathematical Physics in Several Variables", Springer Verlag, 1971. 